\title{
PANORAMio -A Mobile Application BASEd ON ANDROID
}

\author{
Roshan Kavuri ${ }^{1}$, Swetha Pillai ${ }^{2}$, Jacob Sanjay Kumar $^{3}$ Niraj Upadhayaya ${ }^{4}$ \\ , A.Govardan ${ }^{5}$ \\ ${ }^{1,2,3 \& 4}$ Department of CSE \& IT, JB Institute of Engineering \& Technology, \\ Moinabad,Hyderabad,AP,India \\ roshan.kavuriegmail.com \\ ${ }^{5}$ Department of CSE,JNTUH, Hyderabad, AP,India
}

\begin{abstract}
Panoramio is a mobile application which allows the users to find pictures of interesting places with a radar to guide you to them, using GPS. Since the use of smart phones is gaining interest in people, panoramio was implemented as a smart phone application using Google's Android operating system.The application starts by showing a custom map view using mobile maps. The user can pan and zoom the map to select a location. Once a search location has been selected the user taps on a location and the application starts loading all the thumbnails of pictures of all the interesting places taken in that area. By tapping on any thumbnail, information about the particular picture will be displayed. From here the user can view other photos taken by the same user or share the page. And even we can upload these new places. Show your favorite places to the world. Upload your photos to Panoramio. Upload your geolocated photos directly from the Gallery to your Panoramio account which, has following Features includes, Adds a new sharing option for any photo either taken by the built-in camera, or chosen from the gallery, Grab location from EXIF,Allow manual correction of the location.
\end{abstract}

\section{Keywords}

Android, GPS, Mobile maps, Radar, Smart Phone application

\section{INTRODUCTION}

\subsection{Introduction to Panoramio}

Panoramio is an application that enables digital photographers to geo-locate, store and organize their photographs-and to view those photographs in Google Earth and Google Maps.This application where users can upload and geo-locate photos of the world, explore the world through other people's photos, and join a community of other photography enthusiasts. Geo-positioned photos uploaded in Panoramio may be displayed in a Panoramio Group, Google Earth and Google Maps and other sites using the Panoramio API.Panoramio is different from other photo sharing sites because the photos illustrate places. As you browse Panoramio, notice that there aren't many photos of friends and family posing in front of places, or photos of interesting surfaces-Panoramio's all about seeing the world. You can jump from one photo to the closest one, walking virtually around the place or watching the place from many different perspectives. 


\subsection{Existing System}

Today there are many applications and web portals for using maps, one such example is ovi maps. Similarly there are portals to search pictures of interesting places and applications that uses radar to trace routes. But in applications or portals for maps, we can only view and explore the map. In applications or portals of image searching, additional information on location and option to share the information may not be given and in application like radar we can just trace route. But it would be convenient to the common man if all these features come in one single application or portal.

\subsection{Proposed System}

Panoramio extended in order to view a map along with geo-tagging. It would be very convenient if the common man gets the information of all the interesting places at various locations on single portal with pictures and location information. If anyone wants to know about the interesting places at various locations and wants to visit those places then they can get that information through Panoramio.

These are the following features:

- It displays a custom map.

- Displays a list or thumbnails of pictures of the most popular places within the search location.

- Displays the information related to the selected picture.

- Allows information sharing and bookmarking options.

- Allows to view on web

- Can also be used as a radar if the device supports GPS.

\subsection{Modules}

\subsubsection{Map Module:}

The application starts by showing the Google map. As the application starts it shows the world map. On the map graphical user interfaces like zoom buttons are displayed. The user can pan and zoom this map and select a search area. This can be done as follows: the user can pan the map into the direction of the required ord desired location and then when the desired location is on the center of the screen then zoom option can be used to get a detailed view of the map. Panning and zooming is done until the desired location is obtained. After the desired location has been found, it is dragged to the center of the screen and then "Search Panoramio" is button is clicked to view thumbnails of photos of the popular places taken in that area. Thus, in this module the user can view a map, explore and search places.

The google map which is used in the application by adding google API in our eclipse which is the integrated development environment used in the development of panoramio. Then the map API key(MD5 Fingerprint) is to be added to the application code to deploy it. This can be done by submitting the keystore value in the following link http://code.google.com/android/maps-apisignup.html. now when the application successfully runs and on opening shows the map. To this zoom buttons are added to the map using the widgets. Thus, in this module the user can view a map, explore and search places. 


\subsubsection{Search Module}

When the "Search Panoramio" button is clicked the application starts downloading thumbnails of the most popular photos taken within the selected area. After panning and zooming the map until the desired location and is dragged to the center of the screen "Search Panoramio' button is clicked and in a new thread an image list is displayed. The user can select any picture of interest and the pic gets displayed in a separate thread with the author's information. From here when menu is selected four options are shown: Radar, Map, AuthorInfo, View on web. The user can select the "Radar" to trace the route, "Map" to view the location of the photo in the map and "View on web" to navigate to the panoramio site. If the user doesn't use this menu and rather clicks on the selected image, then, again in a new thread an enlarged view of the selected image is. From here when menu is clicked the user gets the options to:

- Add bookmark

- Find on page

- Select text

- Page Info

- Share page

- Download

- Settings

Thus, in this module the user views the image lists, that is, thumbnails, selects desired image, views image's information and then bookmarks and shares their favourite image.

\subsubsection{Radar Module:}

The radar view can be selected once the user selects a picture from the image list. After selecting the picture the application shows the enlarged view of the picture with some additional information. From here when menu is clicked out of three other options, a radar option is found. If radar is selected then the application shows a radar view. But this is possible only if the device on which the application runs supports GPS and radar is installed. Otherwise "NO_RADAR" message is displayed. If the radar is installed and the device on which the application runs supports GPS then the application opens a radar view. In this the latitude values and longitude values are displayed. The gps locates the users current location and then finds and shows the route to selected picture's location in the real world.

Thus in this module the route from the users current location to the selected image's location in the real world is displayed in radar view along with the location's latitude and longitude value.

\subsubsection{Author Info}

AuthorInfo shows the information about the author of a particular photo or image. After an image is selected from the image list another thread opens with the image enlarged and with additional information. From here when menu is clicked along with three other options, a authorInfo button is displayed. When clicked on this the application navigates to the panoramio site and displays a list of other photos taken by the author and also the number of views for each photo. Other than displaying other photo's taken by the author, the author's profile is also displayed with the author's profile pic, message, status, tags, groups and favorite photographs. From here the user has the options to send a private message to the author or add the photo as a favorite photo.

Thus, in this module the user navigates to the author's profile in the panoramio site to view detailed information of the author of a particular image. 


\subsubsection{Web Module:}

Web module allows the user to view the panoramio site. The user can navigate to the site by clicking the "view on web" button. A new thread opens showing the panoramio site. The user can view all photos in the panoramio site, view profiles of different authors/users, add a pic as favorite, share the pic with any other person, bookmark the page etc. the user can upload their photo from their gallery. Thus, this module allows the user to use the panoramio site and allows them to do all the same things that they do in the application but, the difference is that here they are in an online mode and do all the operations directly through the site.

\section{PRILIMINARIES}

Today, there are more mobile computers than there are any other forms of computing devices. Mobile Internet is nowadays ramping up faster than desktop Internet did due to the convergence of five trends: $3 \mathrm{G}$ Internet connection, social networking, video, VoIP and high-end mobile devices. Furthermore, it is expected that mobile devices will become the number one gate to access the Internet within 5 years and the number of mobile devices will be over 10 times the number of fixed devices accessing the Internet $i b i d$.

\subsection{Methods of Gathering Information:}

Because Android is a relatively new product there are not many books available on the subject and the ones released are very general. There is, however, a vast amount of detailed information on internet-official, Google-run websites as well as private, community-run ones. Androidspecific and project-specific information is mainly obtained from internet Mobile map application provides human a convenient way to view the Geographical information. In the temporary age, people typically keep their phone handy for answering calls, sharing information and checking location. It is no doubt that mobile map application is a prevalent and useful application for point of interest to do research. This section reviews some technical related terminologies and studies on some related researches.

\subsection{Technical Review}

\subsubsection{Mobile Computing:}

Mobile computing was born in the early 1990's with the advent of full-function laptop computers and wireless LANs (Garlan, 2002). Mobile Computing is a general term used to describe technologies that enable end-users to access network services any place, anytime, and anywhere. Information access via a mobile device is plagued by lower available bandwidth, unpredictable network quality, and poor security (Stevenson, 2002). Unlike their wired counterparts, design of software for mobile devices must consider resource limitation, battery power and display size. But still, mobile computing is a very active. Mobile computing supports mobile applications, which reduce duplication of data inputs and provide instant information just-in-time. It is now becoming de-facto standard for any IT infrastructure in the current and future system designs.

\subsubsection{Google phone - ANDROID:}

Android is a mobile operating system that uses a modified version of the Linux kernel (Linux, 1991 and Kroah-Hartman, 2010). Android allows developers to develop code in the Java language (Sun Microsystems, 1990), controlling the device via Google-developed Java libraries (Shankland, 2007). Android provides an open development platform and offers developers the 
ability to build innovative applications. Developers take the advantage of the device to access location information, run background services, set notifications to the status bar, etc. The application framework is designed for simply reuse of components. Android employs a set of $\mathrm{C} / \mathrm{C}++$ libraries used by various components of the Android system. These capabilities are exposed to developers through the Android application framework.

\subsubsection{Wireless / Mobile application:}

The fundamental idea behind the wireless network is network connections without wires. In the simplest sense, there are needs from the end-users and together with the growth of technology supports wireless technology. Wired networks are for communication between fixed locations while wireless is for communication between devices; this means we are no longer dependable on the location (Clark, 2008). Wireless technologies cover a wide range, which allow users to establish wireless connections across long distances, unlike infrared light, which is optimized for a short-range wireless connection. Mobile computing applications reduce duplication of data inputs and provide instant information just-in-time. With mobile application,

Companies have more ways to restructure their business processes to realize greater efficiency.

\subsubsection{Google Maps: (Google Inc. 2005)}

It is an application which supports basic web mapping service and technology. It is an open map provided by Google to organize world's information in form of digital image. The main advantages of Google Maps are to make dynamic maps and provide an interactive interface to the user. User can drag the map to fit his/her satisfaction without waiting for a long time.

\subsubsection{Google Mobile Maps: Google Maps}

For mobile was first launched in 2006 (Google Inc. 2006). It enables users to view interactive maps. Google Mobile Maps is an innovative mobile mapping and local search application used widely. It provides the feature like GPS positioning function to mobile phone which has built-in GPS function. Combining Google Mobile Maps and Google Maps API, they offer a number of utilities to manipulate digital maps. Users can add-on some contents on the map through a variety of services.

This newly launched application allows programmers to create. Robust map applications on the mobile. Although Google Maps for Mobile support varieties of rofunctions, it supports a limited number of all cell phones, like Android, BlackBerry, Windows Mobile and iPhone. Its support is somewhat lacking in some of the mobile platform.

\subsubsection{Studies of Global Positioning System (GPS)}

Global Positioning System, formally known as the NAVSTAR - Navigation Satellite Timing and Ranging Global Positioning System (Dana 1999). It is one of the most significant advanced navigation and positioning technology developed recently.

The GPS consists of three main components, which are GPS Ground control stations, GPS satellites and GPS receivers. The ground stations send control signals to the GPS satellites. As depicted in the GPS satellites transmit radio signals and the GPS receivers receive these signals and use it to calculate its position. 


\section{SYSTEM ARCHITECTURE}

Most mobile systems extend an existing business system

or

Interface with an existing system.

There are typically three major components:

- An existing system

- A middleware application

- A handheld application

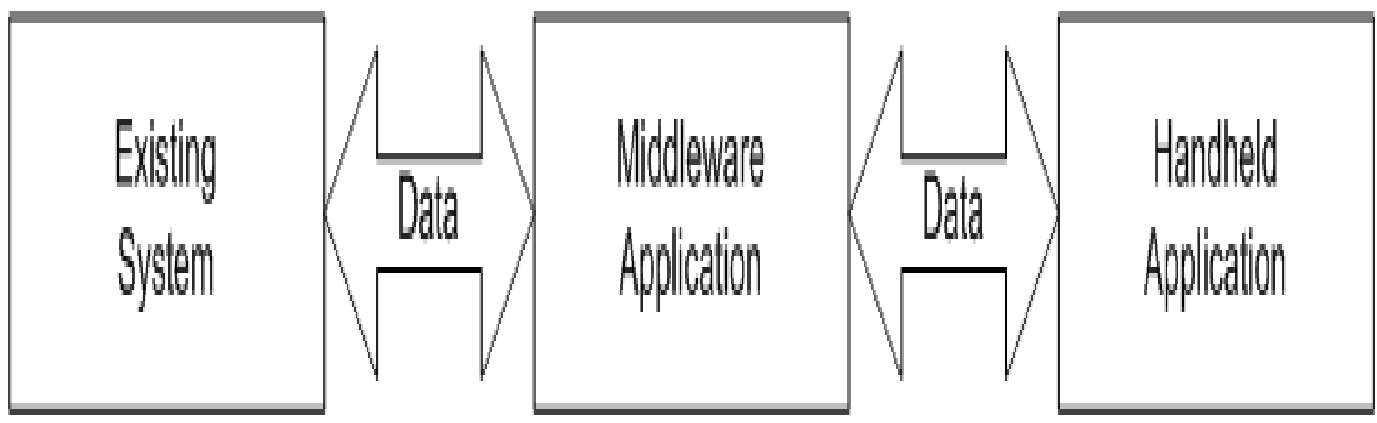

\section{Fig1:system architecture}

The reason a middleware application is usually needed is to provide data transformation, apply business logic, and be a central point of communication for the devices.

If a new business system is being developed or rewritten then no middleware may be necessary; the appropriate logic can be built into the system to communicate with the devices from the start.

In our project, Google Earth is the existing system. We apply a few new or enhanced features to this application and thus create a new application. To Google earth we add thumbnails of photos of the most popular places taken in a particular area.

We add photo sharing and radar features to create a new application. The following is the system architecture for Panoramio application: 


\section{IMPLEMENTATION}

\subsection{Introduction to ANDROID}

The Android platform is a software stack for mobile devices including an operating system, middleware and key applications. Developers can create applications for the platform using the Android SDK. Applications are written using the Java programming language and run on Dalvik, a custom virtual machine designed for embedded use, which runs on linux kernel

top of a

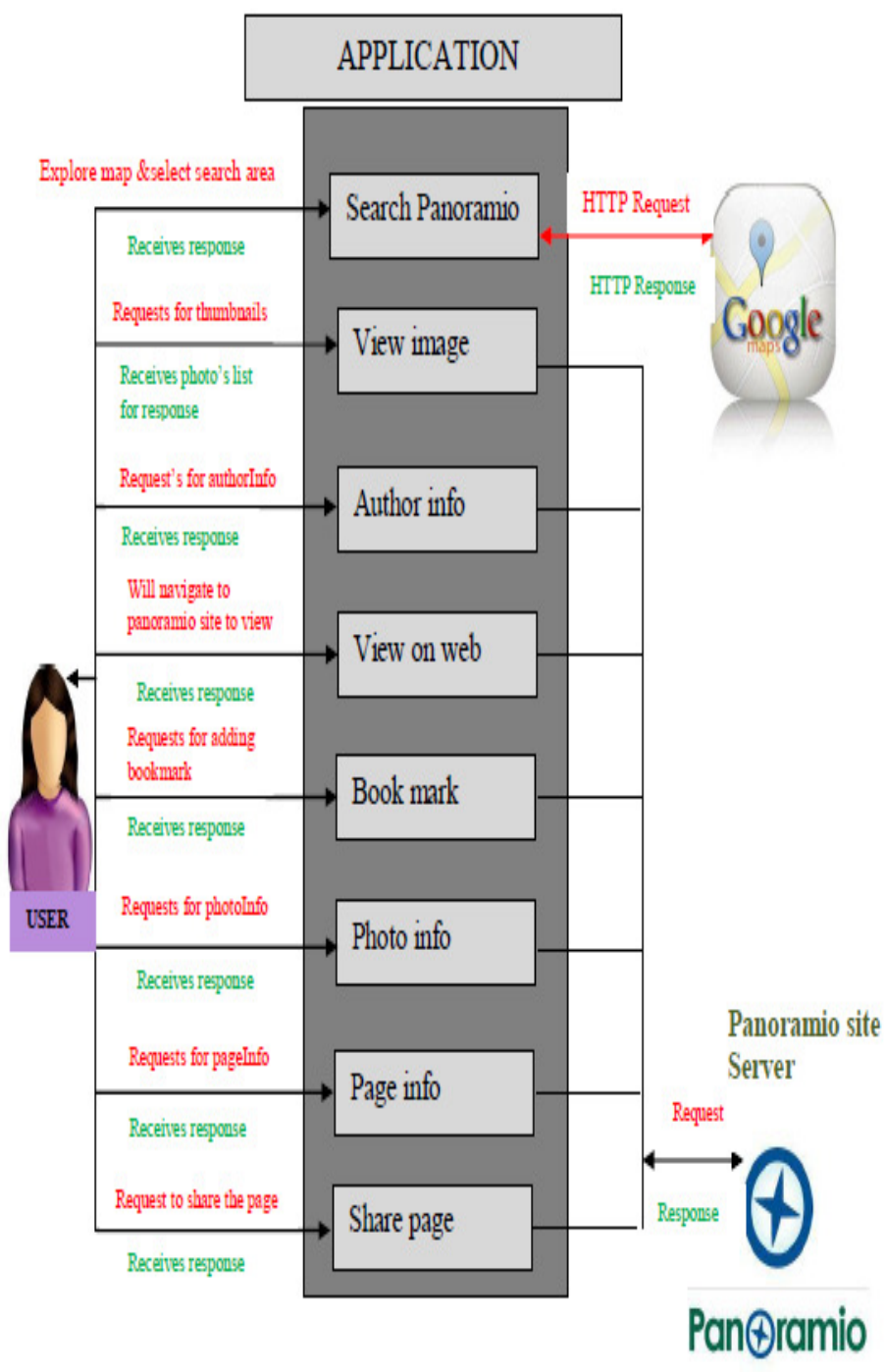

Fig2: Panoramio System Architecture 
4.2 ANDROID Architecture: The following diagram shows the major components of the Android operating system. Each section is described in more detail below.

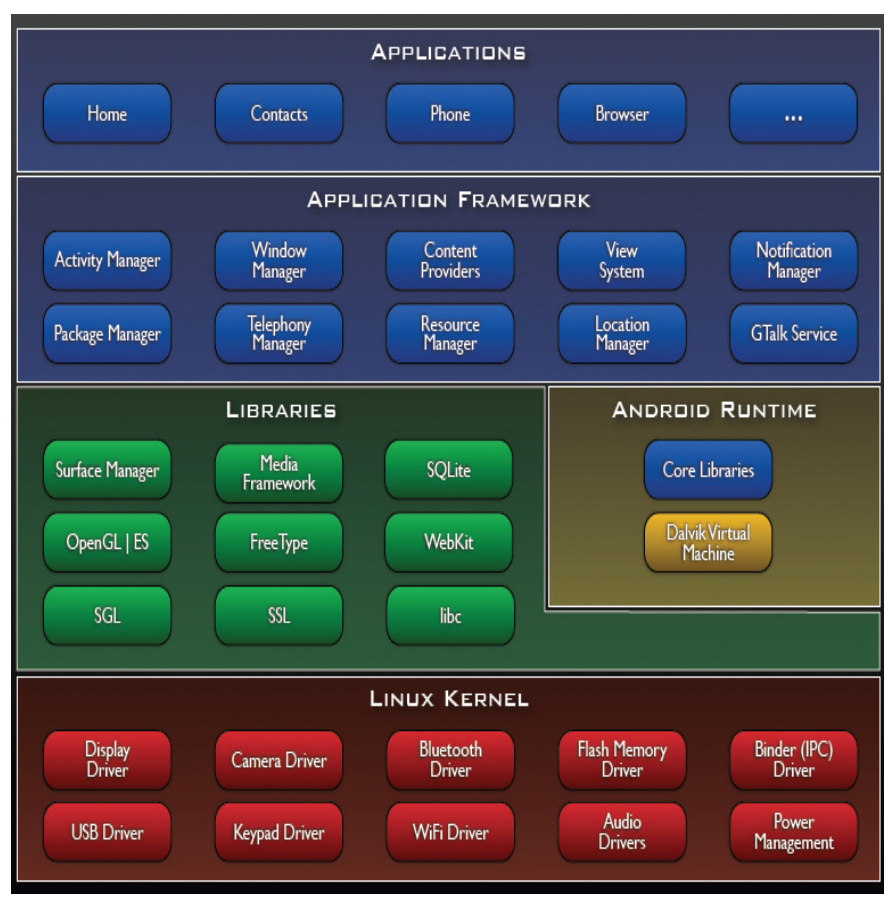

fig:3 Android Architecture

\subsection{Applications:}

Android will ship with a set of core applications including an email client, SMS program, calendar, maps, browser, contacts, and others. All applications are written using the Java programming language.

\subsection{Application Framework:}

Developers have full access to the same framework APIs used by the core applications. The application architecture is designed to simplify the reuse of components; any application can publish its capabilities and any other application may then make use of those capabilities (subject to security constraints enforced by the framework). This same mechanism allows components to be replaced by the user.

Underlying all applications is a set of services and systems, including:

- A rich and extensible set of Views that can be used to build an application, including lists, grids, text boxes, buttons, and even an embeddable web browser

- Content Providers that enable applications to access data from other applications (such as Contacts), or to share their own data

- A Resource Manager, providing access to non-code resources such as localized strings, graphics, and layout files 
- A Notification Manager that enables all applications to display custom alerts in the status bar

- An Activity Manager that manages the life cycle of applications and provides a common navigation back stack

\section{4'4 Libraries:}

Android includes a set of $\mathrm{C} / \mathrm{C}++$ libraries used by various components of the Android system. These capabilities are exposed to developers through the Android application framework. Some of the core libraries are listed below:

- System C library - a BSD-derived implementation of the standard C system library (libc), tuned for embedded Linux-based devices

- Media Libraries - based on PacketVideo's OpenCORE; the libraries support playback and recording of many popular audio and video formats, as well as static image files, including MPEG4, H.264, MP3, AAC, AMR, JPG, and PNG

- Surface Manager - manages access to the display subsystem and seamlessly composites 2D and 3D graphic layers from multiple applications

- LibWebCore - a modern web browser engine which powers both the Android browser and an embeddable web view

- SGL - the underlying 2D graphics engine

- 3D libraries - an implementation based on OpenGL ES 1.0 APIs; the libraries use either hardware 3D acceleration (where available) or the included, highly optimized 3D software rasterizer

- FreeType - bitmap and vector font rendering

- SQLite - a powerful and lightweight relational database engine available to all applications

\subsection{ANDROID Runtime:}

Android includes a set of core libraries that provides most of the functionality available in the core libraries of the Java programming language.

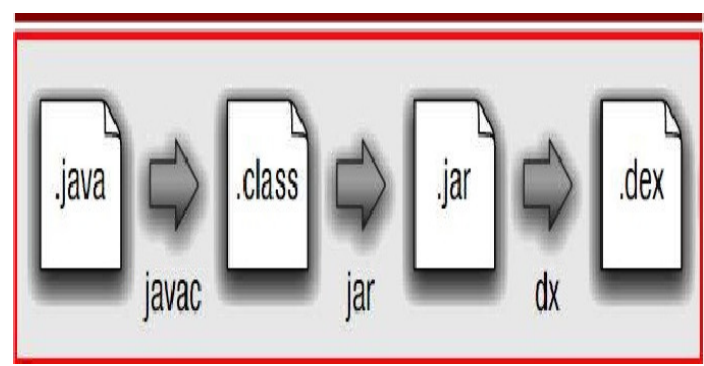

Fig4: conversion of ".java" to ".dex"

Every Android application runs in its own process, with its own instance of the Dalvik virtual machine. Dalvik has been written so that a device can run multiple VMs efficiently. The Dalvik VM executes files in the Dalvik Executable (.dex) format which is optimized for minimal memory footprint. The VM is register-based, and runs classes compiled by a Java language compiler that have been transformed into the .dex format by the included "dx" too The Dalvik VM relies on the Linux kernel for underlying functionality such as threading and low-level memory management. 


\subsubsection{The Key Features of Java is Byte Code:}

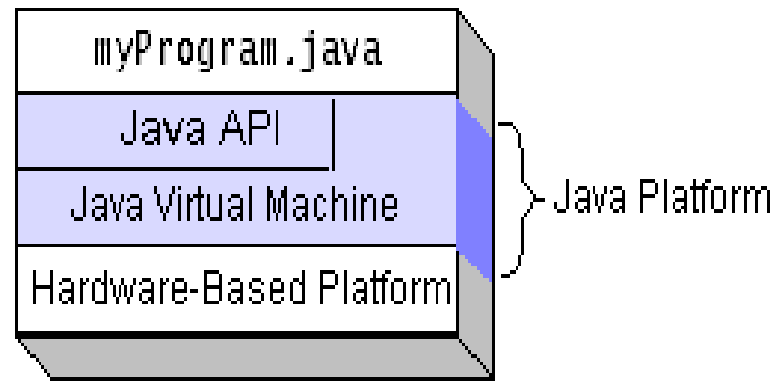

Fig5: java components

The key that allows Java to solve both the security and the portability problems just described is that the output of a Java compiler is not executable code. Rather, it is Byte code. Byte code is a highly optimized set of instructions designed to be executed by the Java runtime systems, which is called the Java Virtual Machine (JVM). That is, in its standard form, the JVM is an interpreter for Byte code. This may come has a bit of surprise

\subsubsection{ANDROID SDK:}

Android SDK includes a comprehensive set of development tools. These include a debugger, libraries, a handset emulator (based on QEMU), documentation, sample code, and tutorials. Currently supported development platforms include x86-based computers running Linux (any modern desktop Linux Distribution), Mac OS X 10.4.8 or later, Windows XP or Vista. Requirements also include Java Development Kit, Apache Ant, and Python 2.2 or later. The officially supported integrated development environment (IDE) is Eclipse (3.2 or later) using the Android Development Tools (ADT) Plugin, though developers may use any text editor to edit Java and XML files then use command line tools to create, build and debug Android applications.

\subsubsection{Widget:}

Widgets are a feature introduced in Android 1.5 and vastly improved in Android 3.0 and 3.1. A widget can display an application's most timely or otherwise relevant information at a glance, on a user's Home screen. The standard Android system image includes several widgets, including a widget for the Analog Clock, Music, and other applications. App Widgets are miniature application views that can be embedded in other applications (such as the Home screen) and receive periodic updates. These views are referred to as Widgets in the user interface, and you can publish one with an App Widget provider. An application component that is able to hold other App Widgets is called an App Widget host. 


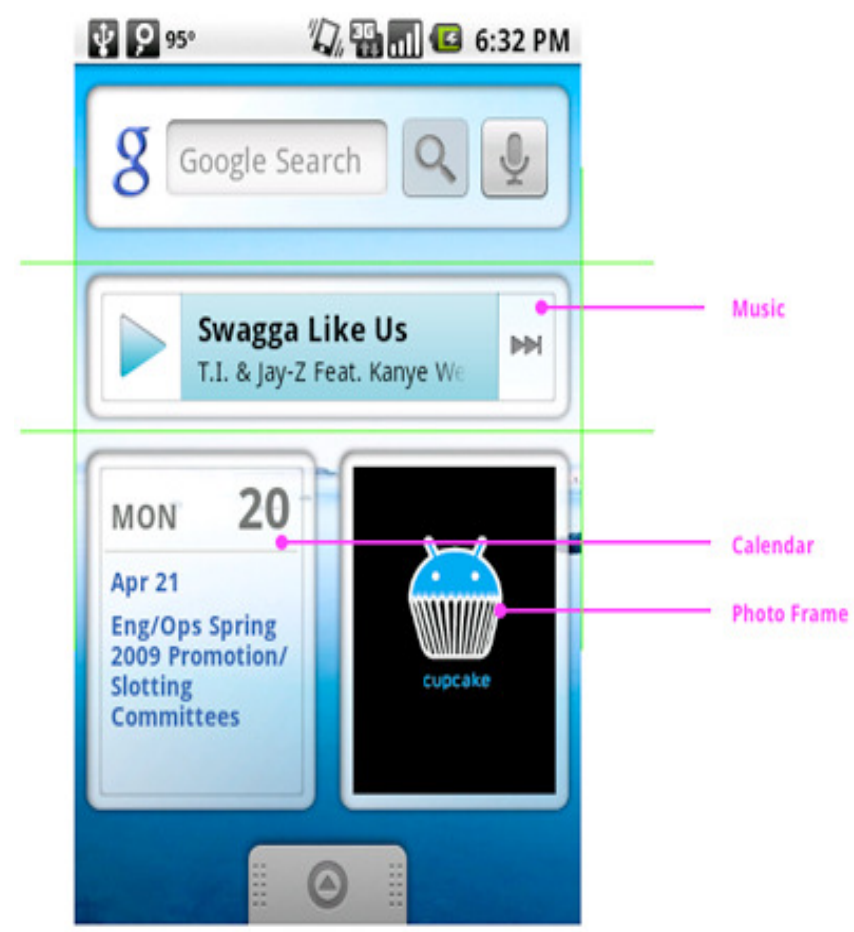

Widgets are a new feature that application developers can use to promote a small sample of the most relevant dynamic data from their applications on the Home screen. We've designed widgets to fit within our Home screen grid framework, which means you and your designer can create a widget within a $4 \times 1,3 \times 3$, or $2 \times 2$ grid cell, depending on the space you need for an at-a-glance summary of information from your application.

\subsubsection{APPLICATION FEATURES}

The application's features are as follows:

- It displays a custom map.

- Displays a list or thumbnails of pictures of the most popular places within the search location.

- Displays the information related to the selected picture.

- Allows information sharing and bookmarking options.

- Allows to view on web

- Can also be used as a radar if the device supports GPS

\section{CONCLUSION}

If you like looking at pictures of places before you get to them, then this application is definitely worth looking into. It's also really interesting to try out the radar feature and get an idea of how that will definitely become more widely used in future apps. 


\section{FUTURE SCOPE}

In future this paper is very useful to communicate with the people in their local languages who are in different countries.

Voice processing feature can be added.

This project can implement the following features:

- Support more than 20 different language pairs.

- SMS

- E-Mail

- Chatting in Android emulator

\section{REFERENCES}

[1] Richardson, L. and S. Ruby, RESTful Web Services2007, Sebastopol, CA: O'Reilly Media, Inc. 446.

[2] Fielding, R., Architectural Styles and the Design of Network-based Software Architectures, in Information and Computer Science2000, University of California, Irvine: Irvine, CA. p. 162.

[3] Fielding, R., et al. Hypertext Transfer Protocol -- HTTP/1.1 (RFC 2616). 1999 5/20/2011]; Available from: http://tools.ietf.org/html//rfc2616.

[4] de la Beaujardiere, J., ed. OpenGIS Web Map Server Implementation Specification, Version 1.3.0. Vol. OGC® 06-042. 2006, Open Geospatial Consortium. 85.

[5] Vretanos, P.A., ed. Web Feature Service Implementation Specification, Version 1.1.0. Vol. OGC 04094. 2005, Open Geospatial Consortium. 117.

[6] Whiteside, A. and J.D. Evans, eds. Web Coverage Service (WCS) Implementation Specification, Version 1.1.0. Vol. 06-083r8. 2006. Open Geospatial Consortium. 129.

[7] Nebert, D., A. Whiteside, and P.P. Vretanos, eds. OpenGIS ${ }^{\circledR}$ Catalogue Services Specification (OGC 07-006r1). Version 2.0.2, Corrigendum 2 Release ed. 2007.

[8] Open Geospatial Consortium, OpenGIS SWE Service Model Implementation Standard, 2011, Open Geospatial Consoritum. p. 155.

[9] The Busy Coder's Guide to Advanced Android Development- By Mark Murphy.Pro Android 3 By Satya Komatineni, Dave MacLean, and Sayed Hashimi.

[10] Developers.android.com

[11] android-developers.blogspot.com

[12] www.adodis.com/Android-Developers.php

\section{About the Author:}

Mr. Roshan Kavuri has obtained his B.E Degree from Andhra University during 1988-92, and M.Tech (CSE) from JNT University, Kukatpally, Hyderabad. He is having nearly 20 years experience in industry as well as a faculty of Computer Science and Information Technology departments. He is pursuing his $\mathrm{PhD}$ from JNTU Hyderabad. His area of research includes Computer Architecture, Parallel Computing, Operating Systems and Computer Networks. Presently he is working as Associate Professor in JB Institute of Engineering Technology, Hyderabad

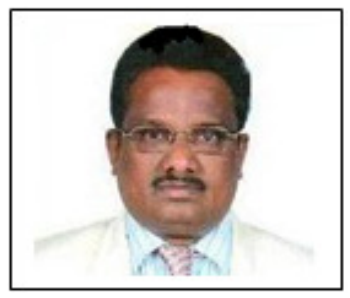
since 2004. 